\title{
Flight Model Development of Tokyo Tech Nano-Satellite Cute-1.7 + APD II
}

\author{
By Hiroki ASHIDA, Junichi NiSHIDA, Kuniyuki OMAGARI, Ken FuJIWARA, \\ Yasumi KondA, Tomio YAMANAKA, Yohei TANAKA, Masaki MAENO, \\ Kota FuJIHASHI, Shinichi InAgaWA, Yoshiyuki MiURA and Saburo MatunaGA \\ Department of Mechanical and Aerospace Engineering, Tokyo Institute of Technology, Tokyo, Japan
}

(Received May 7th, 2008)

\begin{abstract}
The Laboratory for Space Systems at the Tokyo Institute of Technology has developed the nano-satellite Cute-1.7+APD. The satellite was launched by JAXA M-V-8 rocket on February 22, 2006 and operated for about a month. A successor to the Cute-1.7+APD was developed and is named Cute-1.7+APD II. This new satellite is based on its predecessor but has some modifications. In this paper an overview of the Cute-1.7 series and flight model development of Cute-1.7+APD II are introduced.
\end{abstract}

Key Words: CubeSat, CUTE-I, Nano-Satellite, PSLV

\section{Introduction}

The Laboratory for Space Systems (LSS) at the Tokyo Institute of Technology has developed and launched two satellites. The $1 \mathrm{~kg}$ pico-satellite named CUTE-I, which is the first CubeSat in the world, was launched by Eurockot's Rockot launch vehicle in June $2003^{1)}$. CUTE-I was developed in a student-lead program, managed to fully conduct its missions and is still transmitting its house keeping data to the Earth. The flight model of CUTE-I is shown in Figure 1.

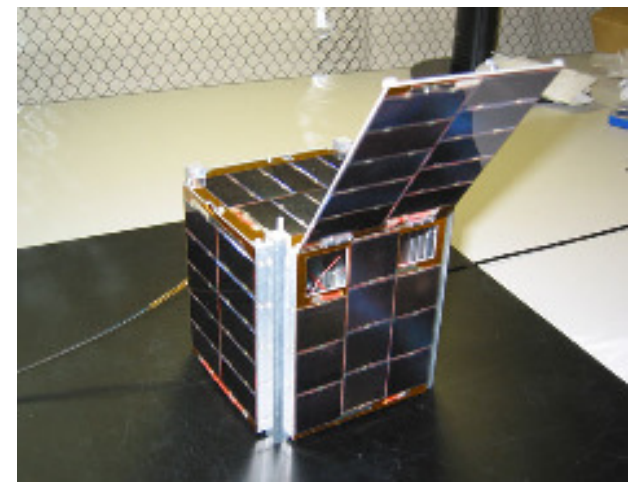

Fig. 1. CUTE-I

Based on this satellite development procedure and acquired nano-satellite bus technology, the LSS began its next satellite project, "Cute-1.7+APD", pursuing innovation in small satellite systems. This satellite was launched by JAXA M-V-8 launch vehicle on February 22, 2006, and had operated for a little more than a month ${ }^{2}$. After achieving success in some of the missions the satellite stopped replying to any uplink commands from the ground station at Tokyo Tech. Cute-1.7+APD is now transmitting a continuous wave that is not modulated. While the recovery operation has been continuing for a year, the problem from its housekeeping data has been thoroughly investigated and a ground experiment has concluded that it was the result of a kind of a space radiation hazard. Figure 2 shows the flight model of Cute-1.7 + APD.

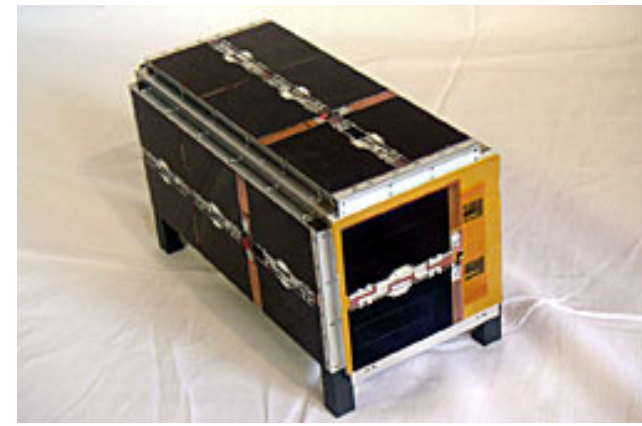

Fig. 2. Cute-1.7+APD

The "Cute-1.7+APD II" project, the successor to the Cute-1.7 + APD, began in April 2006 to complete the missions which Cute-1.7+APD was, unfortunately, not able to accomplish ${ }^{3)}$. Cute-1.7+APD II is essentially a modification of Cute$1.7+\mathrm{APD}$, with changes not only to prevent the radiation hazard but also to conduct the missions more effectively. This satellite is now in India and waiting for the launch by Indian rocket PSLV-C9 which is scheduled to be launched at the end of April 2008.

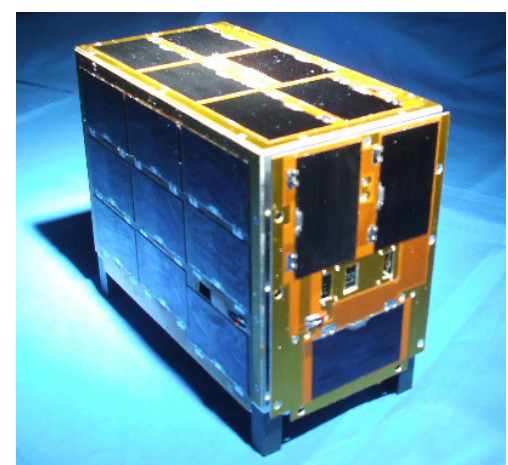

Fig. 3. Cute-1.7+APD II

In this paper an overview of the Cute-1.7 series, modifications, some special features of Cute-1.7+APD II and its current status are introduced. 


\section{Overview of the Cute-1.7 Project}

In this chapter the objectives and missions of the Cute1.7+APD series are introduced.

\subsection{Objectives}

The Cute-1.7+APD project has two objectives.

The first objective is to facilitate future micro-satellite development projects by demonstrating a new design methodology. The methodology, aiming toward rapid and lowcost development, includes proactive use of high performance and low cost commercial devices in orbit. The Cute-1.7+APD series are equipped with several COTS (Commercial Off-TheShelf) devices including widely used PDAs (Personal Digital Assistants) as their OBC (On-Board Computer) and amateur radio transceivers as communication devices.

The secondary objective is to provide flight experiment opportunities to space engineering researchers and students by developing a highly versatile satellite. To this aim, this satellite contains an engineering mission shared with researchers in the field of control engineering. On-board devices for attitude determination and control such as three magnetic torquers and a software upload function which can update software inside the PDAs from the ground enable on-orbit experiments in advanced attitude control algorithms. In addition, the Cute-1.7 was designed to provide other researchers an opportunity to demonstrate their devices using a simple interface between the satellite bus system and themselves. A scientific observation device, APD (Avalanche Photo Diode) sensor, is implemented on the Cute-1.7+APD series to demonstrate it in orbit.

\subsection{Missions}

In an effort to search for a rapid and low-cost satellite design, one mission was assigned to demonstrate the use in space of PDAs as the primary computers in this satellite. This approach comes from advances in PDA technology which have resulted in low cost, high computing performances and a wide range of software development environments. In addition to these functional advantages another objective is to acquire the necessary engineering knowledge to adjust commercial devices to a space environment. For one, the Cute-1.7+APD series uses two PDAs which can be switched over in case of malfunction for the purpose of redundancy. The PDA used in the satellites is shown in Fig. 4.

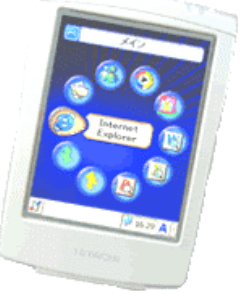

Fig. 4. Hitachi NPD-20JWL

Cute-1.7+APD II will also conduct three missions for the purpose of providing space experiment opportunities using nano-satellites.

One is a three-axis attitude control experiment using the magnetic torquers whereby the satellite's control algorithms can be demonstrated. A conceptual diagram is shown in Fig. 5.

In another experiment the satellite demonstrates a new APD sensor, developed by the Laboratory for Experimental AstroPhysics at the Tokyo Institute of Technology, to observe the density of low energy charged particles in LEO (Low Earth Orbit). This sensor is planned to be used in the LSS's next satellite as a part of a scientific observation device after passing this flight experiment ${ }^{4}$. Fig. 6 shows the APD sensor.

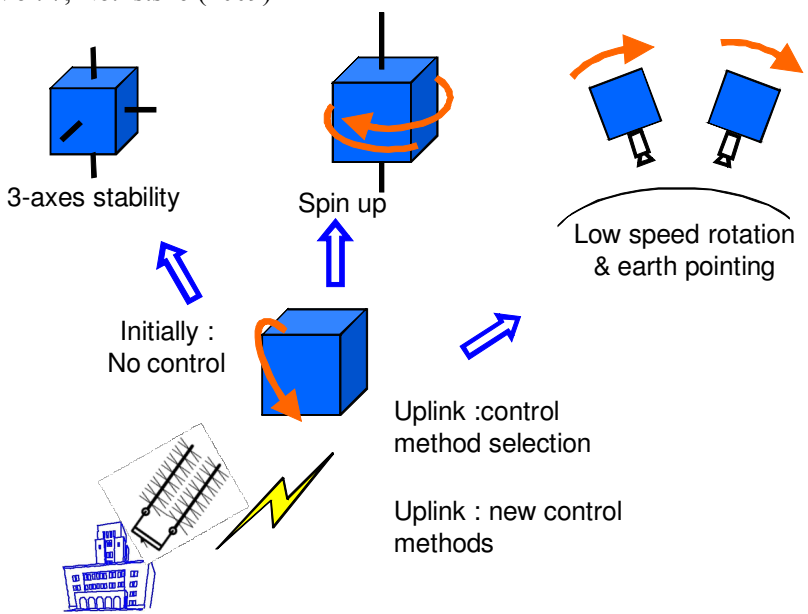

Fig. 5. Attitude Control Mission

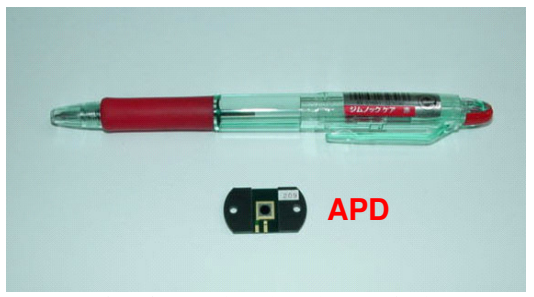

Fig. 6. Avalanche Photo Diode

This satellite also provides a digital repeater function which is a public massage box to ham operators. By using this function ham operators can upload their massage to this satellite and the massages can be downlinked in a certain period. This enables them to exchange messages with not only close operators but also distant operators such as an operator who is on the other side of the Earth.

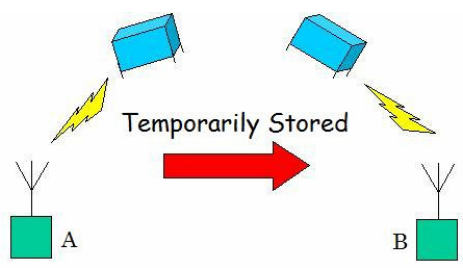

Amateur Radio Stations

Fig. 7. Amateur Service Mission

In order to expand the use of nano-satellites in space, Cute1.7+APD is also managed to conduct an experiment in deorbit technology using electrodynamic tethers. Based on the concern over space debris from inactive satellite, deorbitting technology composed of small components can be an important factor for the future of nano-satellite development. As a first step for the development of this technology, this satellite deploys $10 \mathrm{~m}$ of an electrodynamic tether. This mission, shown in Fig.8 was conducted only in Cute-1.7+APD because the orbit of Cute1.7+APD II is too high to conduct the deorbitting experiment.

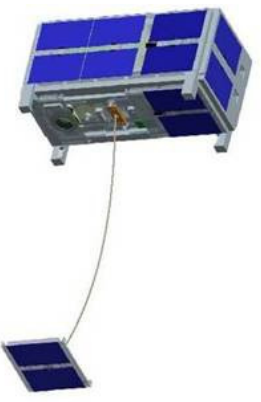

Fig. 8. Tether Deployment Mission 


\section{Development of Cute-1.7 + APD II}

In this chapter the configurations, the results of environmental tests and the current status of the Cute-1.7 + APD II are introduced.

\subsection{Configurations}

Figure 9 shows the functional diagram of Cute-1.7+APD II. Thirteen blocks out of the total thirty employ commercial products including PDAs, memory cards, USB (Universal Serial Bus) hubs, digital cameras and handheld amateur radio transceivers. Most subcomponents are connected to a PDA through the USB interface that enables plug and play. The connectivity contributes to designing each subcomponent as a module and expanding versatility of this satellite by providing easy connection to various mission devices.

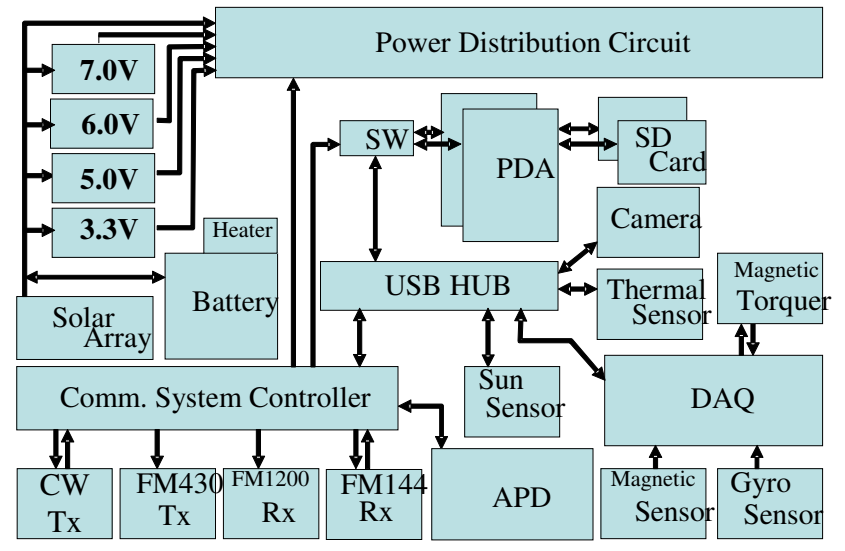

Fig. 9. System Block Diagram of the Cute-1.7 + APD II

The development of the Cute-1.7 + APD II was advanced by categorizing it into nine subsystems: ADCS (Attitude Determination and Control Subsystem), C\&DH (Command and Data Handling), Communication, EPS (Electrical Power Subsystem), Camera, Structure, Separation System, Ground Station and Science (APD). Details of each subsystem are described below.

(1) Attitude Determination and Control

As the satellite is very small and has been developed within the university, small-size and low-cost sensors have been employed. Although they have an advantage of size and cost, their accuracy is not high enough to satisfy the mission demands. Consequently, the system is constructed using a combined sensor system that is composed of multiple attitude determination sensors, as shown in Fig. 10: sun sensors, magnetic sensors and gyroscopes. In addition to these, advanced attitude determination algorithms such as the QUEST method were implemented to improve the accuracy of the attitude determination.



S6560



ADXRS150

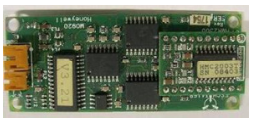

HMR2300
Fig. 10. Attitude Determination Sensors

(Left: Sun sensor, Center: Gyroscope, Right: Magnetic Sensor)

S6560, which is manufactured by Hamamatsu Photonics, has two photo-diodes per unit whereby a difference of currents between them indicates, via a known correlation, the incident angle of light. They, therefore, were used as sun sensors. Two units each are mounted on five of the sides except for the top side of the satellite. The sensor's coverage is $84 \%$ of the whole sky.
The Analog Devices' ADXRS150 was used as a gyroscope. It can measure an angular velocity around a single axis and output the measured value as analog data. This analog data is acquired by an A/D converter of a DAQ (Data Acquisition Device) controller. The temperature of the gyroscope is also determinable and enables appropriate calibration of the instrument to compensate for temperature dependency.

HMR2300, produced by Honeywell, was used as the magnetic sensors. It consists of Honeywell's subcomponent of magnetic sensors (HMC1001 and HMC1002) and a digital interface. A performance test was conducted inside a magnetic shield with the support of JAXA.

Data from these sensors is gathered by the PDA and used for the calculations for attitude determination or to downlinking to the ground station.

The satellite has four algorithms for attitude determination: QUEST, REQUEST, EKF (Extended Kalman Filter) and UF (Unscented kalman Filter). The use of a high-performance PDA enables a real-time attitude determination of the satellite using these advanced algorithms. An attitude estimation will also be done on the ground by recalculating using the sensor data from the satellite and its result will be used to confirm the result of on-orbit attitude determination experiment by comparing them.

The control actuator employs a magnet torquer, shown in Fig. 11 , for three axis attitude control. Improvements were made so that an output torque of the actuator is enlarged by dividing a coil into three segments and connecting them in parallel, which leads to an increase in its consumption of the current. Specifications of the magnetic torquer are shown in Table. 1.

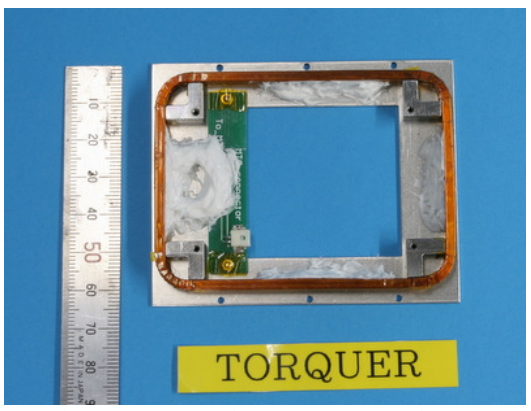

Fig. 11. Magnetic Torquer (1 Axis)

Table. 1. Specification of the Magnetic Torquer

\begin{tabular}{|c|c|}
\hline Type & Air Core Coil \\
\hline Dimensions & $58.5 \times 78.3 \times 1.6\left[\mathrm{~mm}^{3}\right]$ \\
\hline Weight & $5[\mathrm{~g}]$ \\
\hline Resistance & $180[\mathrm{ohm}]$ \\
\hline Power Consumption & $900[\mathrm{~mW}]$ \\
\hline Output Magnetic Dipole & $0.15\left[\mathrm{Am}^{2}\right]($ per coil) \\
\hline
\end{tabular}

(2) Command and Data Handling

The primary MPU consists of two PDAs for redundancy. Hitachi's NPD-20JWL, shown in Fig. 4 and Table 2, was selected because of its small-size which can be put inside the two-unit CubeSat $\left(100 \times 100 \times 200 \mathrm{~mm}^{3}\right)$.

Table. 2. Specification of the PDA

\begin{tabular}{|c|c|}
\hline Model & NPD-20JWL \\
\hline Manufacturer & Hitachi \\
\hline Dimensions & $70 \times 100 \times 5\left[\mathrm{~mm}^{3}\right]$ \\
\hline Weight & ARV4I 400MHz \\
\hline CPU & Microsoft Windows CE.NET \\
\hline OS & 32 MByte] \\
\hline RAM & SD Card 128 [MByte] \\
\hline Flash Memory & USB, MMC/SD Card Slot \\
\hline Interfaces & \\
\hline
\end{tabular}

Data that cannot be handled by the PDA such as analog data is gathered by the DAQ controller. By the commands from the PDA, it acquires the analog data, configures a data frame and 
sends it to the PDA through a USB interface. Figure 12 shows a block diagram of the C\&DH subsystem.

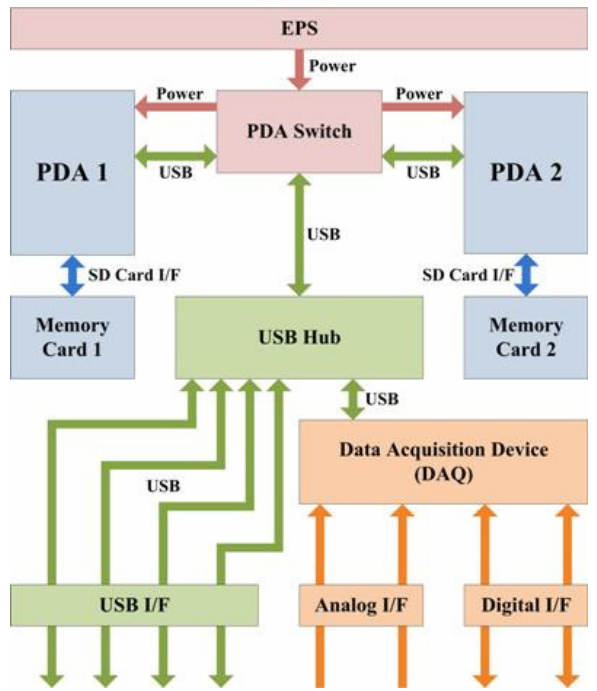

Fig. 12. C\&DH Subsystem Block Diagram

\section{(3) Communication}

The communication module has four channels; $144 \mathrm{MHz}$ AFSK (Audio Frequency Shift Keying) for a command uplink, 1.2GHz GMSK (Gaussian filtered Minimum Shift Keying) for a public message uplink, $430 \mathrm{MHz} \mathrm{CW}$ (Continuous Wave) for a beacon downlink and $430 \mathrm{MHz}$ AFSK and GMSK for a packet downlink.

Four monopole antennas were used for these four bands. The antennas were made of teflon-coated iron steel to provide insulation, environmental resistance and attenuation.

As the communication subsystem is a minimum configuration of the satellite, it has responsibility for a crisismanagement. Two communication controllers monitor all important conditions of the satellite, e.g. bus voltages, and power off or reset a device when the satellite is in danger.

The transceiver circuit and the software inside the communication controller were improved far more reliable than Cute-1.7+APD.

(4) Electrical Power

The EPS uses $4 \mathrm{Li}$-ion batteries, shown in Fig. 13, in parallel and $33 \mathrm{GaAs}$ solar cells that generate a $4 \mathrm{~W}$ power supply on average. The EPS provides four stabilized buses; $3.3 \mathrm{~V}, 5 \mathrm{~V}, 6 \mathrm{~V}$ and $7 \mathrm{~V}$ for various components of the satellite.

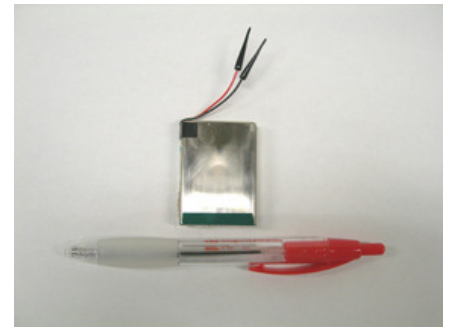

Fig. 13. Li-Ion Battery

The EPS has two important modifications from that of the Cute-1.7+APD.

First is a radiation tolerance. In the first satellite, because of the SEL (Single Event Latch-up) occurred in the microcontroller of the communication subsystem, the satellite became unable to conduct all missions. The alternative is to use a current monitoring circuit than can detect a SEL and restart the component when it occurs. The circuit showed effective tolerance to radiation in a ground experiment. In addition, the microcontroller can mutually monitor the other one and both microcomputers can reset each other when one of them stops working.
Another modification was to increase the power generation of the small satellite. The orbital operation showed relatively low power compared to the estimation, which affected the satellite's operation procedures. The power generation is enhanced by increasing the dimension of the satellite.

(5) Camera

The Cute-1.7+APD II has a compact CMOS camera module which is designed for a mobile phone. The camera module is controlled by Renesas' $\mathrm{H} 8$ microcontroller and its data is temporally stored in a FIFO (First Input, First Output) memory before it is sent to the PDA via USB as shown in Fig. 15. A sample picture taken by the on-board camera is shown in Fig. 16.

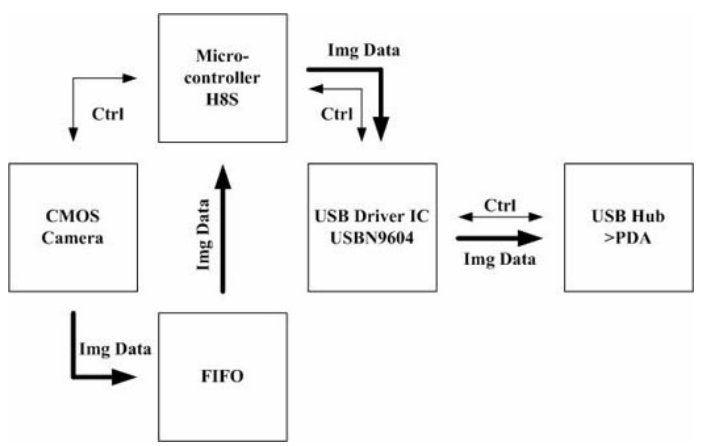

Fig. 15. Camera Subsystem Block Diagram

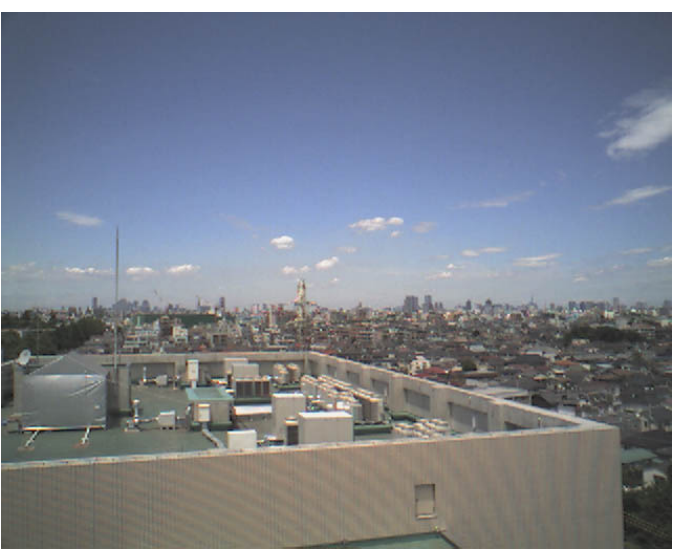

Fig. 16. Picture Taken by the On-Board CMOS Camera

\section{(6) Structure}

Cute-1.7+APD II measures 115 x 180 x $220 \mathrm{~mm}^{3}$ and weighs $3 \mathrm{~kg}$. The main structure of the satellite is made of aluminium. The satellite is about 1.5 times larger than the former satellite to increase its power generation as described in Section (4). In addition to this, the structural change also contributes to the assembly process. As many missions were installed to the former satellite, the high density of components required a long time to assemble them. A mother board which has an external access port also helps assembling and debugging.

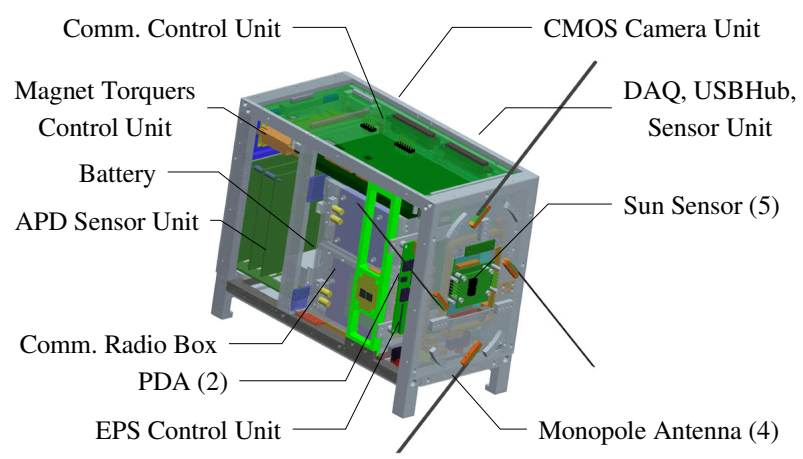

Fig. 17. Components Layout 


\section{(7) Separation System}

The LSS's separation system, shown in Fig. 18, has been demonstrated for three times in orbit and has been proven very reliable system. The satellite's leg is tightly held by four latch levers of the separation system during a launch, and the latch levers are tied with two nylon strings, as shown in Fig. 19. When the separation signal is given by the launch vehicle the circuit of the separation system begins a count-down. After waiting for a given duration the heat wire inside the separation system is heated to cut the nylon string. Then the finger rotates as a result of a torsion spring so that it removes itself from the notch on the pillars of the satellite. Finally the spring inside the separation system pushes the satellite up and releases it from the launch vehicle.

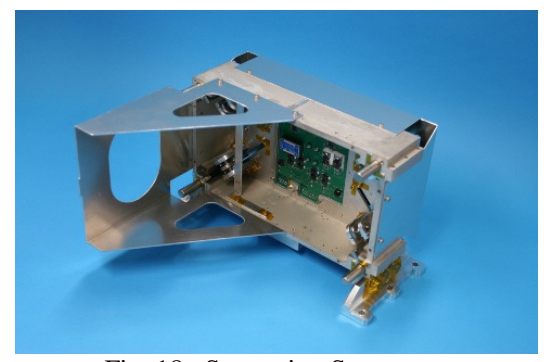

Fig. 18. Separation System

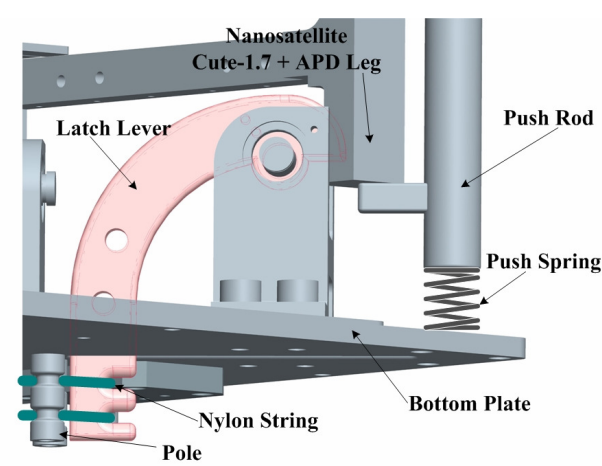

Fig. 19. Holding Mechanism

\subsection{Results of Tests}

Many environmental tests had been conducted on the Cute1.7+ APD II to make sure it will work well in orbit.

\section{(1) Vibration Test}

A vibration test was conducted with the support of JAXA. The level of the vibration was the QT (Qualification Test) Level of the PSLV. From an appearance and electrical check, it is confirmed that the satellite is tolerable for the predicted vibration during the launch.

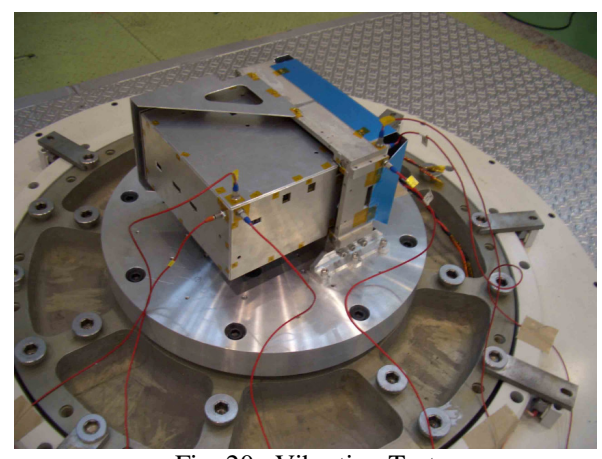

Fig. 20. Vibration Test

(2) Radiation Test

As it was expected that the microcontroller of the communication subsystem had stopped working in the former satellite because of a radiation hazard, a radiation test was

conducted at the Research Center for Nuclear Physics at the Osaka University. When protons, which have energy of $60 \mathrm{MeV}$, were emitted against the microcontroller an SEL frequently occurred and large-currents were detected. This result led the satellite to have an over-current monitoring circuit per one microcontroller.

(3) Thermal Test

The severe temperature changes were expected because one third of an expected orbit of the Cute-1.7+APD II was umbra. Therefore, a thermal test was conducted by using the LSS's thermostatic chamber for the subcomponents and the whole satellite, and confirmed the satellite's nominal behaviour for -20 to 60 degree Celsius.

(4) Vacuum Test

Antenna deployment, high voltage application to the APD module and demonstrations of other devices under vacuum condition were totally successful.

(5) Attitude Determination and Control Simulation

A simulator for the attitude determination and control, shown in Fig. 21, was made to conduct ground experiments to demonstrate their algorithms. MATLAB on the computer calculates an orbit of the satellite and obtains pseudo values of the attitude sensors. Orbital calculations includeing perturbations and magnetic fields were calculated by using the IGRF (International Geomagnetic Reference Field) model.

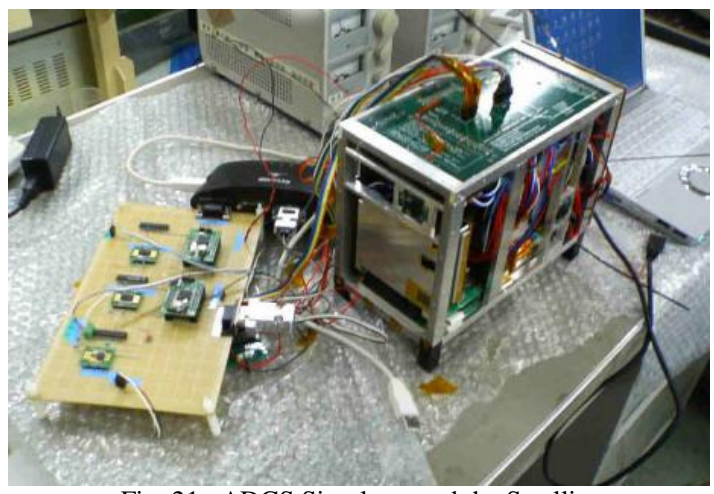

Fig. 21. ADCS Simulator and the Satellite

\subsection{Current Staus}

Cute-1.7+APD II was shipped to India via Canada. In April a launch campaign team went to India to conduct final checks of the satellite and confirmed its perfect performance as shown in Fig. 22. The satellite was assembled to the launch vehicle, as shown in Fig. 23, and was ready for launch.

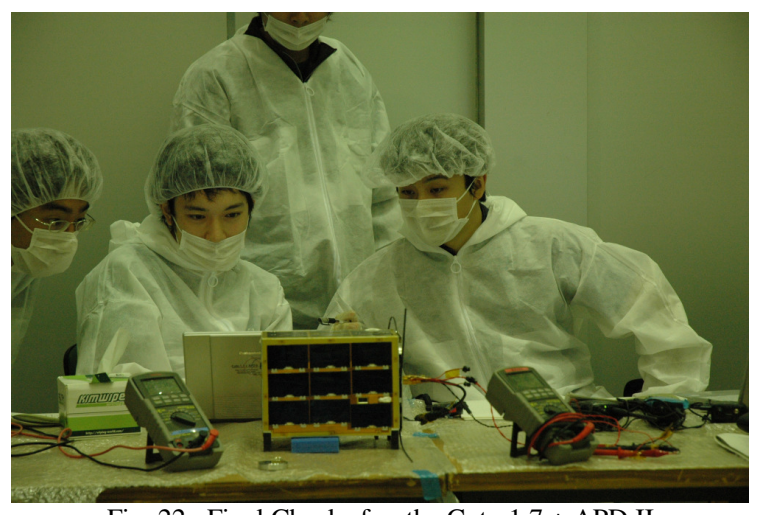

Fig. 22. Final Checks for the Cute-1.7 + APD II 


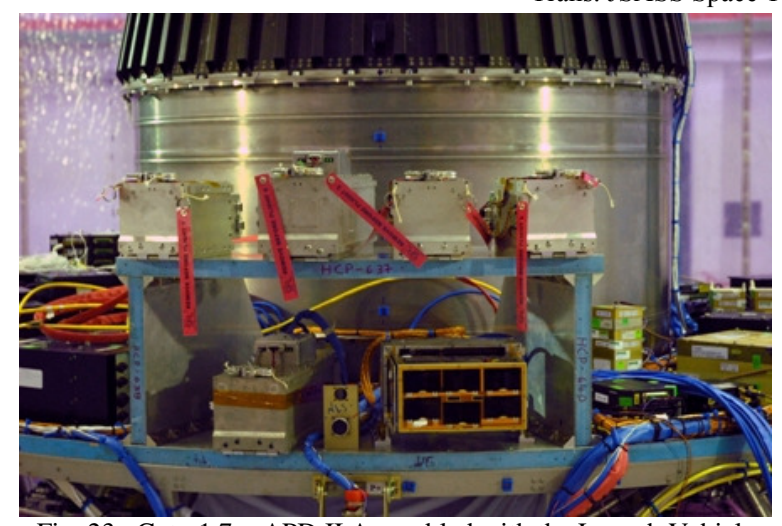

Fig. 23. Cute-1.7 + APD II Assembled with the Launch Vehicle

The satellite was successfully launched into space together with 6 university satellites in an Indian rocket, Polar Satellite Launch Vehicle (PSLV) as shown in Fig. 24. Its orbit is a circular sun-synchronous with an altitude of approximately $630 \mathrm{~km}$ and inclination of about 98 degrees. Cute-1.7+APD II is now at the initial operation phase and are being checked the basic functions such as power generation and communication.

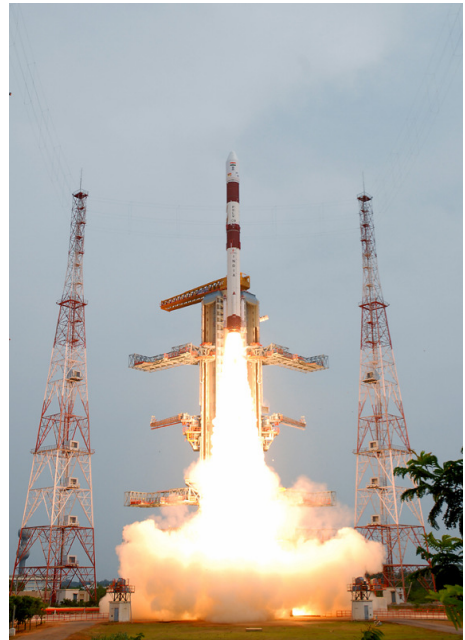

Fig. 24. Launch of the PSLV-C9 (Courtesy of ISRO)

\section{Conclusion}

The development of the $3 \mathrm{~kg}$ pico-satellite Cute-1.7+APD II was introduced. The configurations and modifications of the onboard devices are described. In addition to this, many environmental tests and attitude simulations were conducted throughout its development to make sure that the satellite can accomplish its missions whilst withstanding space environments such as space radiation. It was launched into orbit, and is in the initial operation term. The missions will be conducted in orbit as soon as the initial operation has been completed.

The development was finished and with the experiences obtained by the development of the satellite. The LSS just has began to develop its next small satellite which will conduct novel engineering and scientific missions.

\section{References}

1) Nakaya, K., Konoue, K., Sawada, H., Ui, K., Okada, H., Miyashita, N., Iai, M., Urabe, T., Yamaguchi, N., Kashiwa, M., Omagari, K., Morita, I. and Matunaga, S.: Tokyo Tech CubeSat: CUTE-I - Design \& Development of Flight Model and Future Plan -, AIAA 21 st International Communications Satellite Systems Conference and Exhibit, Yokohama, 2003, AIAA 2003 2388.

2) Miyashita, N., Iai, M., Omagari, K., Imai, K., Yabe, H., Miyamoto, K., Iljic, T, Usuda, T., Fujiwara, K., Masumoto, M., Konda, Y., Sugita, S., Yamanaka, T., Konoue, K., Ashida, H. and Matunaga, S.: Development and Flight Report of PicoSatellite Cute-1.7 + APD, 25th International Symposium on Space Technology and Science, Kanazawa, June 4-11, 2006, 2006-f-08.

3) Fujiwara, K., Omagari, K., Iljic, T., Masumoto, S., Konda, Y., Yamanaka, T., Tanaka, Y., Maeno, M., Ueno, T., Ashida, H., Nishida, J., Ikeda, T., and Matunaga, S.: Tokyo Tech NanoSatellite Cute-1.7 + APD Flight Operation Results and The Succeeding Satellite, The 17th IFAC Symposium on Automatic Control in Aerospace, Toulouse, June 25-29, 2007.

4) Nishida, J. and Tsubuku, Y.: Tokyo Tech's Technology Demonstration Satellite "TSUBAME", $21^{\text {st }}$ Annual Conference on Small Satellite Student Competition, Utah, 2007

5) Maeno, M., Omagari, K., Iljic, T., Masumoto, S., Fujiwara, K., Konda, Y., Yamanaka, T., Tanaka, Y., Ueno, T., Ashida, H., Nishida, J., Hagiwara, Y., Fujihashi, K., Ikeda, T., Inagawa, S., Miura, Y. and Matunaga, S.: Development of Tokyo Tech NanoSatellite Cute-1.7 + APD II, The 17th Workshop on JAXA Astrodynamics and Flight Mechanics, Sagamihara, 2007

6) Omagari, K., Miyashita, N. and Matsunaga, S.: Development, Launch and Operation of Tokyo Tech's Nanosatellite Cute-1.7 + APD, The 16th Workshop on JAXA Astrodynamics and Flight Mechanics, Aug 1-2, 2006, Sagamihara, pp.209-214

7) Konda, Y., Usuda, T., Sagami, T., Omagari, K., Kashiwa, M. and Matunaga, S.: Development of Attitude Determination and Control System for Pico-Satellite Cute-1.7 + APD, The 16th Workshop on JAXA Astrodynamics and Flight Mechanics, Aug 1-2, 2006, Sagamihara, pp.242-247 